\title{
POSTER
}

\section{Prise en charge des tumeurs brunes en chirurgie orale : étude d'un cas et conduite à tenir}

\author{
Robardey $G^{\star}$, Samama $M^{\star \star}$, Lescaille $G^{\star}$, Descroix $V^{\star}$, Goudot $P^{\star \star}$ \\ *Service d'Odontologie, CHU Pitié-Salpêtrière, Paris \\ ${ }^{*}$ Service de Chirurgie Maxillo-Faciale, CHU Pitié-Salpêtrière, Paris
}

Mots clés : tumeur brune, hyperparathyroïdie, diagnostic, conduite à tenir, mandibulaire, maxillaire, lésion ostéolytique, granulome à cellules géantes

\section{Introduction}

La tumeur brune est une lésion ostéolytique bénigne rare résultant d'une anomalie du métabolisme osseux dans l'hyperparathyroïdie (HPT), pouvant toucher les os de la face. Si la prise en charge des HPT est consensuelle, celle des tumeurs brunes porte à discussion (Reséndiz-Colosia J. A. et al. Head Neck 2008 30:1497-1504).

Ce cas décrit le diagnostic et la prise en charge chirurgicale de tumeurs brunes mandibulaires et fait le point sur les stratégies thérapeutiques.

\section{Observation}

Une patiente de 82 ans, avec pour antécédents une insuffisance rénale chronique terminale dialysée et une HTP secondaire, consultait pour gêne prothétique. L'examen clinique retrouvait une mobilité de la branche horizontale droite sans hypoesthésie dans le territoire du nerf maxillaire inférieur, une discrète voussure vestibulaire mandibulaire droite, l'absence de douleur spontanée ou provoquée. L'orthopantomogramme montrait des lésions radioclaires multilobées mandibulaires bilatérales évocatrices d'ostéolyse et une probable fracture parasymphysaire droite sans déplacement, confirmée par la tomodensitométrie. Une biopsie a été réalisée et l'examen anatomo-pathologique retrouvait un granulome central à cellules géantes. Le diagnostic de tumeur brune a alors été évoqué et confirmé en présence de l'HTP secondaire traitée et partiellement contrôlée. Compte tenu de la fracture pathologique et du contexte, une prise en charge chirurgicale de type énucléation kystique bilatérale sous anesthésie générale avec mise en place d'une plaque d'ostéosynthèse, a été décidée.

\section{Discussion}

L'intérêt de ce cas réside dans la description de la démarche diagnostique et de l'indication chirurgicale dans le cadre d'une tumeur brune symptomatique sur un terrain d'HPT secondaire à une insuffisance rénale chronique.

Le chirurgien oral peut en effet être amené à diagnostiquer une tumeur brune dans deux cas : exploration d'une lésion ostéolytique faisant découvrir une HPT primaire (ce qui est devenu extrêmement rare de nos jours du fait des bilans biologiques de routine) ou sur un terrain d'HPT secondaire connue. Les examens complémentaires n'étant pas spécifiques, le diagnostic ne peut 
être posé que sur une triade biologique, radiologique et histologique (Triantafillidou $\mathrm{K}$ et al. Am J Otolaryngol 2006 27:281-286).

La prise en charge thérapeutique d'une tumeur brune est multidisciplinaire et doit toujours commencer par le traitement étiologique de l'HPT, principalement chirurgical pour les HPT primaires et médical pour les HPT secondaires, qui permet dans la grande majorité des cas une involution de la tumeur (Qaisi M et al. Case Reports in Medicine 2015 ID 567543) en 1 à 20 mois. Cependant, la chirurgie d'énucléation est indiquée d'emblée dans les cas de lésions symptomatiques (compression nerveuse, fracture, déformation) ou à l'origine de troubles fonctionnels invalidants, ou dans un second temps en cas ou d'involution insuffisante de la tumeur voire d'évolution. L'efficacité du traitement endocrinologique doit toujours être réévaluée. 